\title{
LOS OBJETOS DE ADORNO-COLGANTES DURANTE EL SOLUTRENSE EN LA PENÍNSULA IBÉRICA
}

\section{Solutrean personal ornaments in the Iberian Peninsula}

\author{
Bárbara Avezuela Aristu ${ }^{1}$ y Esteban Álvarez-Fernández ${ }^{2}$
}

Recibido el 8 de septiembre de 2013. Aceptado el 17 de octubre de 2013

Resumen. Las excavaciones arqueológicas llevadas a cabo en diferentes yacimientos de la Península lbérica con niveles atribuidos al periodo Solutrense (p. ejp. Las Caldas, La Garma A, Chufín o Aitzbitarte IV en la región cantábrica; Caldeirão, Vale Boi, Buraca Grande en Portugal; Reclau Viver, La Cueva de Ambrosio, Santa Maira o Nerja en la costa mediterránea) cuentan entre sus materiales con un gran número de objetos de adorno-colgantes, sobre todo realizados sobre conchas de moluscos marinos y dientes. Este trabajo sintetiza la información disponible sobre este tipo de objetos en la Península lbérica y los somete a diferentes tipos de análisis: arqueozoológico, tecnológico, tafonómico, funcional, etcétera.

Palabras clave: objetos de adorno-colgantes, Paleolítico superior, Solutrense, Península Ibérica, Europa.

Abstract. The archaeological excavations undertaken at different sites in the lberian Peninsula with levels attributed to the Solutrean (e. g. Las Caldas, La Garma A, Chufin or Aitzbitarte IV in Cantabrian Spain; Caldeirão, Vale Boi, Buraca Grande in Portugal; Reclau Viver, La Cueva de Ambrosio, Santa Maira or Nerja in the Spanish Mediterranean coast) provided us a large number of personal ornaments made mainly from marine shells and teeth. This study synthesizes the available information about Solutrean personal ornaments in the Iberian Peninsula. The study has been carried out from a number of different analytical perspectives: archaeozoological, taphonomic, technological, functional, etc.

Keywords: personal ornaments, Upper Palaeolithic, Solutrean, Iberian Peninsula, Europe.

\section{INTRODUCCIÓN}

Las evidencias de adornos-colgantes más antiguas documentadas en la Península lbérica -exceptuando algunos ejemplos controvertidos como los bivalvos perforados de cronologia musteriense de la cueva de Los Aviones (Zilhão et al. 2010) - remontan al periodo Auriñaciense con elementos elaborados por el Homo sapiens sapiens (ÁlvarezFernández 2010; Álvarez-Fernández y Jöris 2007; Tejero Cáceres 2013). Auriñaciense y Gravetiense presentan una continuidad respecto a las materias primas utilizadas, téc- nicas de fabricación y contactos a media y larga distancia entre diferentes zonas del SO de Europa (Álvarez Fernández y Avezuela Aristu 2012). Es a partir de la época que nos ocupa, el Solutrense, que el número de objetos de adornocolgantes aumenta su número considerablemente en la Península Ibérica, sobre todo en la región mediterránea. En este artículo se recogen los datos disponibles sobre los yacimientos solutrenses de la Península lbérica (Fig. 1) divididos en tres apartados correspondientes a tres zonas geográficas: región cantábrica, Portugal y región mediterránea. Las piezas proceden tanto de excavaciones antiguas así como de exca-

(1) Laboratorio de Estudios Paleolíticos. Dpto. de Prehistoria y Arqueologia, Facultad de Geografía e Historia, Universidad Nacional de Educación a Distancia. Paseo Senda del Rey, 7. E-28040 Madrid (España)·b.avezuel@gmail.com

(2) Dep. Prehistoria, $H^{\mathrm{H}}$ Antigua y Arqueología, GIR PREHUSAL. Fac. de Geografía e Historia. Universidad de Salamanca. Cerrada de Serranos S/N E-37002 Salamanca (España) estebanalfer@hotmail.com·epanik@usal.es 
vaciones más recientes y su estudio ha sido realizado desde diferentes puntos de vista como el tafonómico, arqueozoológico o tecnológico con el fin de hacer una aproximación a distintos aspectos relativos a la selección y aprovisionamiento de materias primas, la movilidad de los grupos y otras cuestiones ligadas al comportamiento humano.

\section{REGIÓN CANTÁBRICA}

Los datos proporcionados en este estudio sobre los objetos de adorno solutrenses de la región cantábrica proceden de la información recogida en la Tesis Doctoral de uno de los firmantes (Álvarez-Fernández 2006; Álvarez-Fernández 2010-2011; Álvarez-Fernández y Fernández 2011). Parte de los materiales fueron recuperados en excavaciones antiguas y están depositados en diferentes museos de la geografía española. El resto pertenecen a excavaciones más recientes Ilevadas a cabo a partir de los años '70, algunas de las cuales siguen vigentes hoy en día.

En esta zona el número de piezas estudiado asciende a 239 adornos (Fig. 2) procedentes de 19 yacimientos (Fig. 1).
La materia prima más utilizada son los dientes de animales $(42,2 \%)$, seguida muy de cerca por las conchas de moluscos marinos (38,5\%). Y en tercer lugar por objetos elaborados en hueso $(11,7 \%)$. El marfil, el asta y las materias primas de origen mineral reducen su existencia a casos testimoniales.

Los dientes más representados son los caninos atróficos de ciervo, Cervus elaphus, (cerca del 76\%), presentes en yacimientos como Las Caldas, el Mirón y Bolinkoba. Los dientes de otras especies también se transforman en objetos de adorno-colgantes pero en un número muy inferior. De este modo encontramos incisivos de ciervo en Amalda, de cabra (Capra pyrenaica) en La Riera, de bóvido en Chufín y de caballo (Equus ferus) en Altamira y El Cierro. Aunque mucho menos frecuentes, están presentes en Altamira caninos de zorro (Vulpes vulpes), de león de las cavernas (Felix spelaea), lince (Felis lynx) y de lobo en La Garma A (Canis lupus). Un diente de delfín mular se ha documentado en el último yacimiento citado.

Entre los moluscos, las conchas de los gasterópodos son las más empleadas; la especie Littorina obtusata la más abundante $(56,8 \%)$ seguida de Trivia sp $(17,6 \%)$ y de Nucella lapillus (15,6\%). Las dos primeras se documentan en yaci-

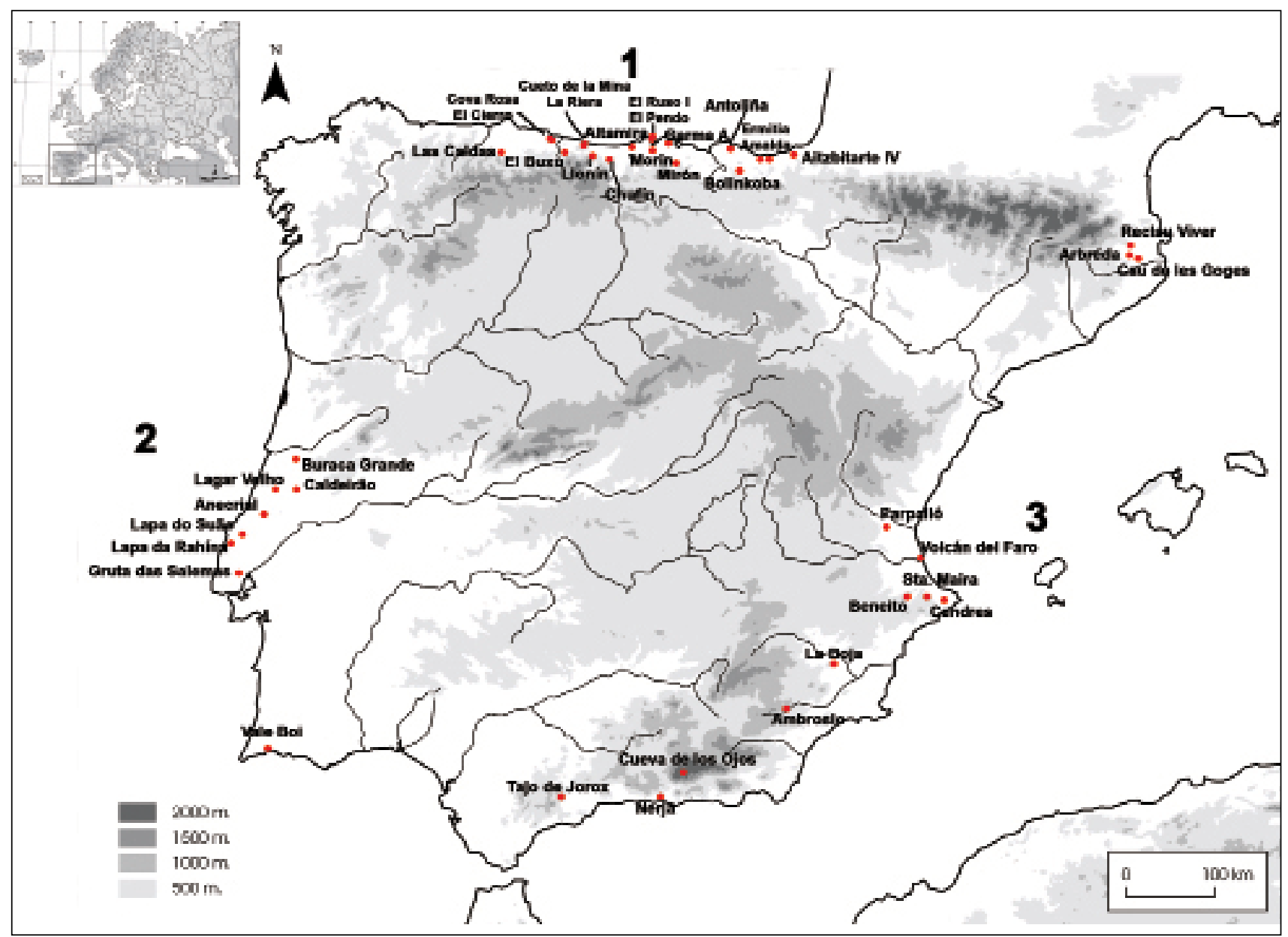

A Figura 1. Mapa de la Península Ibérica con los yacimientos de cronología solutrense con objetos de adorno-colgantes. 1: región cantábrica. 2: Portugal. 3: región mediterránea. 


\section{Región cantábrica ( $\mathrm{n=239)}$}

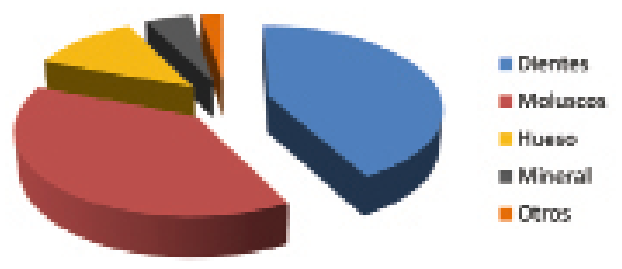

\section{Portugal $(n=117)$}

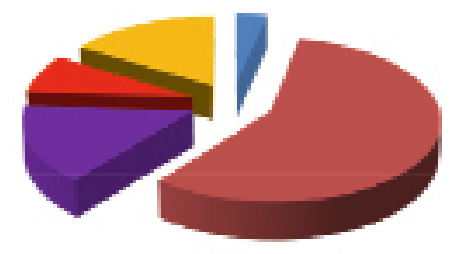

\section{mientes}

EGasterdpodos marinos

Excatbeodes

mGasterbpodos furioles

Hueso

\section{Región mediterránea $(n=3076)$}

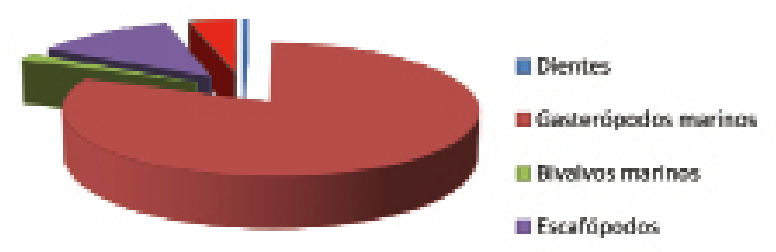

Easterbodos thatsies

A Figura 2. Porcentajes de las diferentes categorias de adornoscolgantes según la materia prima sobre la que están elaborados y por regiones.

mientos como La Riera, El Buxu, El Mirón y Amalda. Otras especies están representadas por un solo ejemplar. El el yacimiento que muestra un conjunto más variado de estas es La Riera (L .obtusatta, N. lapillus, Littorina littorea y Colus sp.). Un ejemplar de la especie mediterránea Nassarius mutabilis se ha documentado en El Ruso I (Fig. 3). En algunos yacimientos, como en La Riera, se han documentado ejemplares de gasterópodos sin valor bromatológico sin perforar (en este caso, L. obtusata). Han sido considerados como materia prima para la elaboración de objetos de adorno-colgantes.

En la región cantábrica los bivalvos perforados no son muy abundantes y se reducen a cinco valvas con una única perforación en la zona del umbo. Están presentes en los yacimientos de Aitzbitarte IV (Laevicardium oblongum y Glycymeris sp.) y Cueto de la Mina (Anadara sp.).

Los escafópodos conforman el 36,2\% del total de moluscos marinos usados como objetos de adorno-colgantes

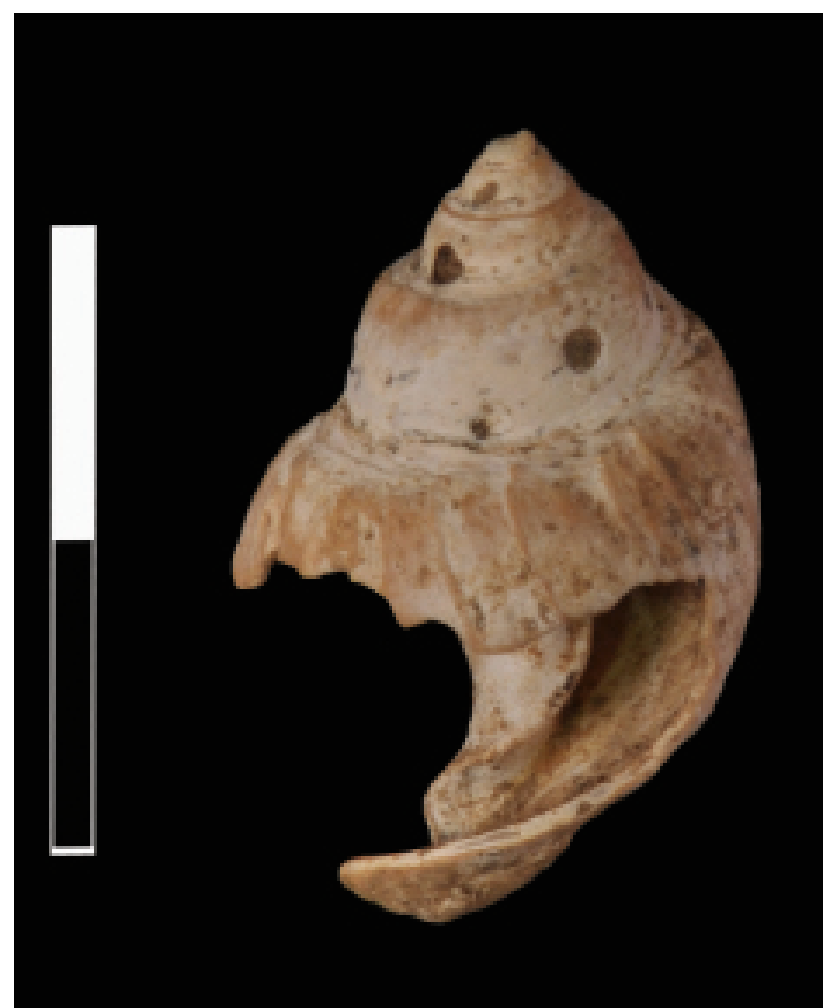

ム Figura 3. Nassarius mutabilis. El Ruso I, nivel III.

empleando siempre su abertura natural como método de suspensión.

También hay piezas elaboradas en otras materias primas de origen animal, como el hueso, por ejemplo, sobre diáfisis de pájaros o de pequeños mamíferos (Cova Rosa), huesos hioides (Altamira) o vértebras de peces (Las Caldas). En materiales mucho menos frecuentes como el asta encontramos dos adornos, uno en la Riera y la llamada Venus de El Pendo (Fig. 5). Existen tres piezas elaboradas en marfil (Las Caldas y La Riera).

Entre las materias primas de origen mineral se documentan perlas confeccionadas en azabache (Las Caldas) y ámbar (Cova Rosa) (Fig. 6). Cantos de cuarcita (Cueto de la Mina) y de goethita (Altamira) con perforaciones naturales pudieron

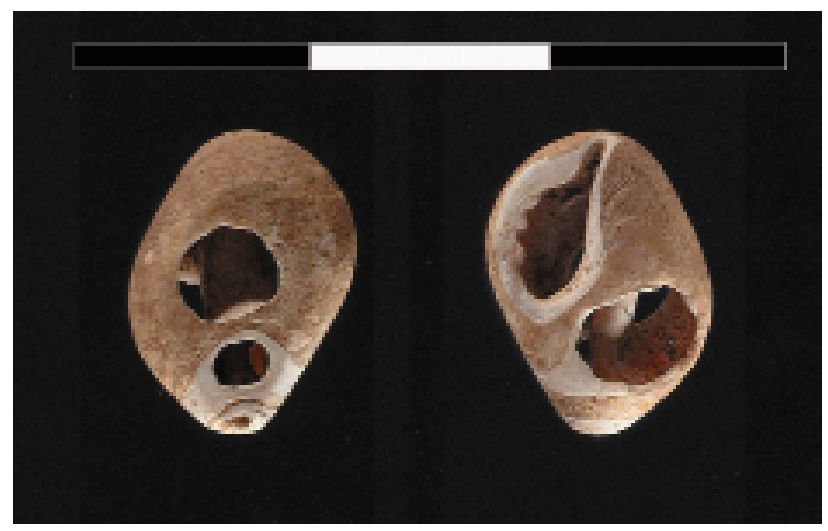

A Figura 4. Nucella lapillus perforada. La Garma A, nivel G. 


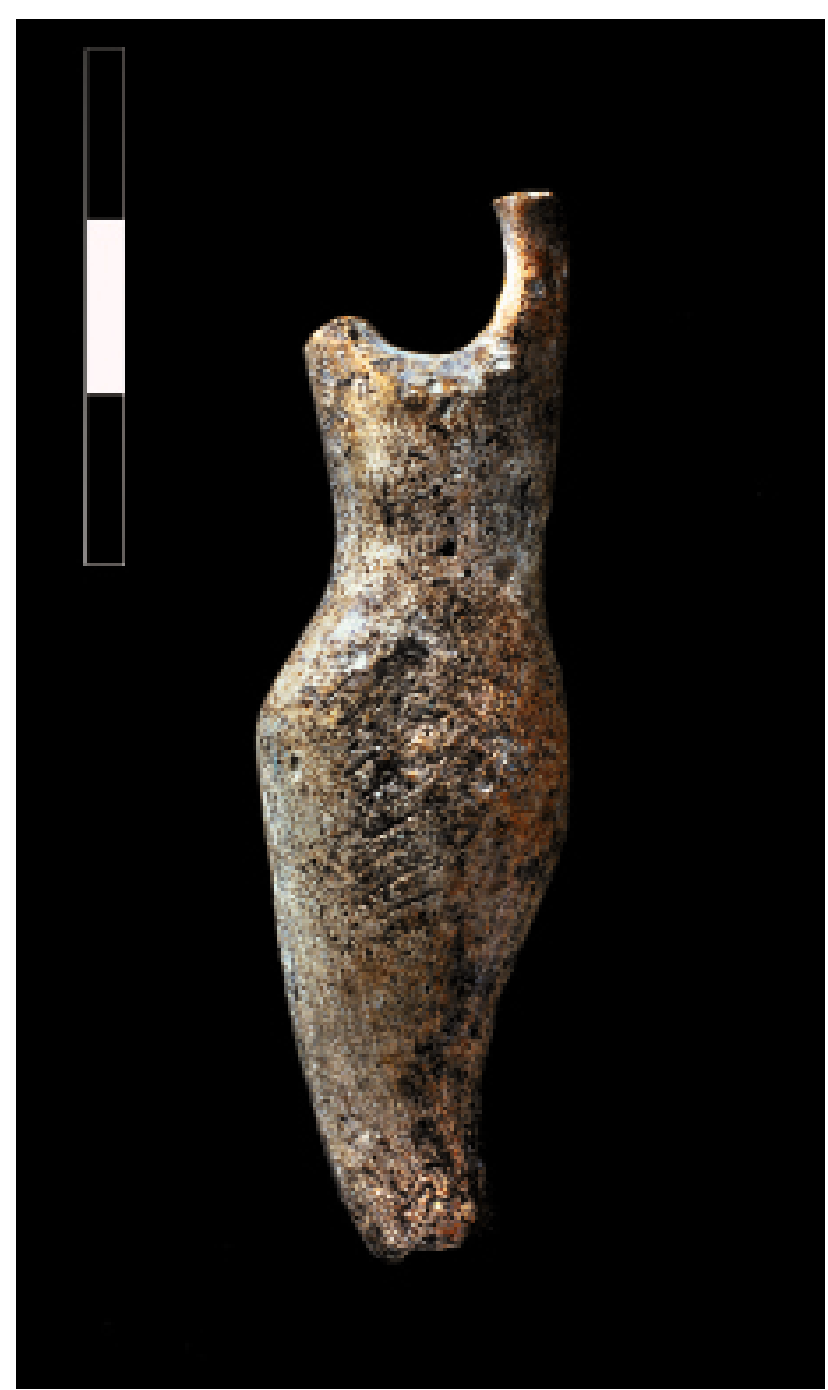

A Figura 5. Asta de cérvido. "Venus". El Pendo.

usarse como objetos de adorno-colgantes. También hay que destacar un diente fósil de tiburón de Aitzbitarte IV (Fig. 7)

Por lo que respecta a cómo fueron modificadas todas estas materias primas para su suspensión se registran varias técnicas. En el caso de los dientes, casi todas las piezas aparecen perforadas en la raíz (perforaciones bicónicas). Gran parte de ellos conservan huellas de la preparación de la superficie a perforar (incisiones profundas, abrasión intensa, etc.).

La mayor parte de los ejemplares de moluscos perforados poseen una sola perforación (75\%), pero también se encuentran ejemplos con dos sobre Trivia (Cova Rosa) y sobre $N$. lapillus (Amalda). Las conchas con tres orificios son excepcionales (uno en La Riera y cuatro en Amalda, todos sobre L. obtusatta de grandes dimensiones). La técnica más usada para obtener estas perforaciones es la abrasión (Fig. 4), pero también se emplean otras técnicas como la percusión, la presión, el serrado o la rotación.

En el caso de los escafópodos siempre se hace uso de su forma tubular natural para llevar a cabo la suspensión, la modificación que se documenta en yacimientos como El Mirón es el serrado del extremo apical, lo que facilita su enfilado.

Sobre las piezas elaboradas en otras materias primas de origen animal y en las de origen mineral, lo más común es la perforación bicónica, con o sin preparación previa de las superficies a horadar. Destaca el modo de suspensión del diente de tiburón de Aitzbitarte IV, cuya raíz posee una ranura y dos muescas laterales.

Algunos de los adornos están decorados con incisiones. Se trata sobre todo de dientes, fundamentalmente caninos atrofiados de ciervo (por ejemplo, en La Riera). La decoración más común son series de pequeñas incisiones paralelas en los laterales. Este mismo tipo de decoración está presente en otros dientes, como el canino de lobo de La Garma A, así como en los huesos hioides de Altamira y en las placas de marfil de Las Caldas. Hay que señalar también que un $15 \%$ de las piezas conservan restos de ocre en sus superficies.

En la región cantábrica los adornos solutrenses pertenecientes, sobre todo, a excavaciones antiguas se recogen en niveles de ocupación en los que no se documentan evidencias que los relacionen con el resto de materiales arqueológicos. Existen algunas excepciones, sobre todo en excavaciones más recientes. Se trata de agrupaciones de objetos de similares características (materia prima, técnicas de

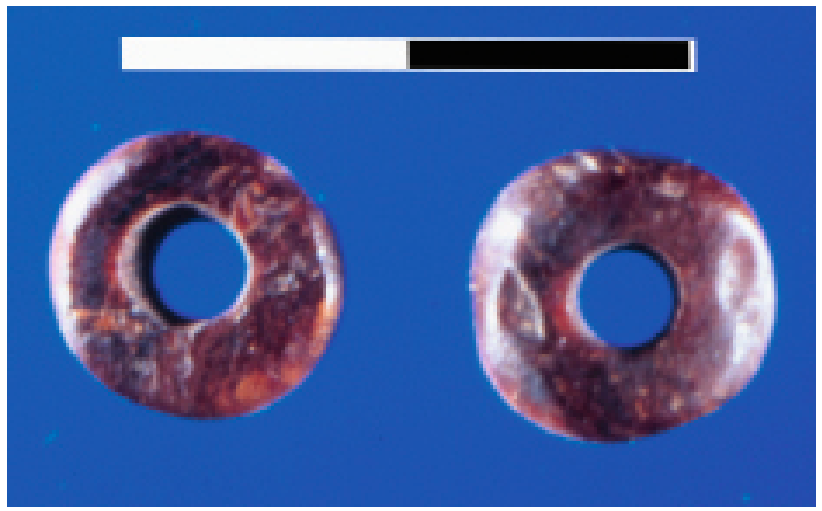

$\Delta$ Figura 6. Cuentas de ámbar. Cova Rosa, Capas 6a $-8^{\mathrm{a}}$.

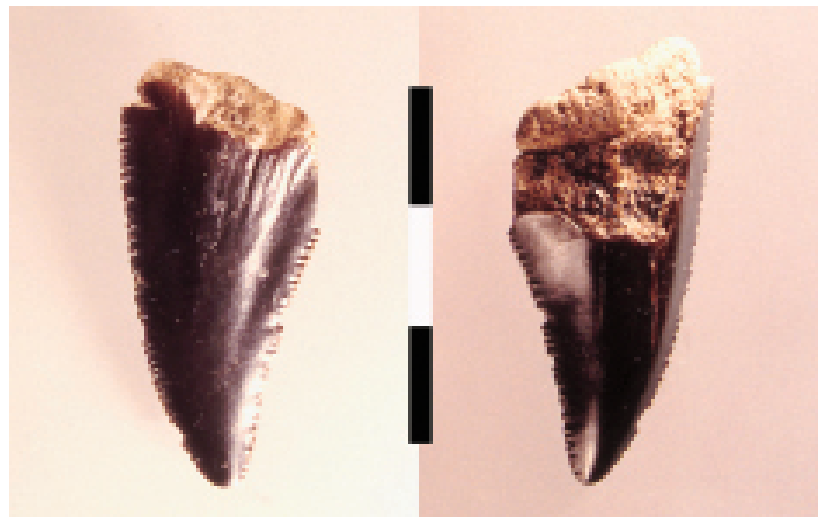

A Figura 7. Diente de tiburón fósil. Aitzbitarte IV, Nivel IVa. 
confección, huellas de uso...) que fueron olvidados, abandonados o depositados intencionalmente. Este es el caso de las cuatro placas elaboradas sobre el hueso estilohioideo de un hioides de caballo procedentes de las excavaciones de Breuil y Obermaier en Altamira o de otros conjuntos similares documentados en El Mirón o La Riera. En el Solutrense medio de Las Caldas también se encuentra un depósito formado por siete caninos atrofiados de ciervo, dos de estas siete piezas pertenecen al mismo animal. Lo mismo ocurre con otro par de caninos atrofiados de ciervo del Nivel 18 de La Riera.

Los datos de que disponemos sobre la manufactura de los objetos de adorno-colgantes, son, sobre todo, aquellos relativos a los objetos fracturados y a la discriminación de las fracturas de uso de aquellas ocasionadas a la hora de elaborar los adornos (incisivos de cabra en La Riera, caninos atrofiados de ciervo en Las Caldas o un canino de lobo en La Garma A), así como aquellas piezas en proceso de elaboración como los tubos de ave de Las Caldas.

\section{PORTUGAL}

En Portugal las evidencias de elementos de adornocolgantes solutrenses se concentran en ocho yacimientos (Fig. 1): Buraca Grande (Moura y Aubry 1994), Lagar Velho (Vanhaeren y D'Errico 2002), Caldeirão (Chauvière 2002), Anecrial (Vanhaeren y D'Errico 2002), Lapa do Suão (Zilhao 2007), Lapa da Rahínha y Gruta das Salemas (Veiga Ferreira y Roche 1980) y Vale Boi (Bicho et al. 2010; Tátá Dos Anjos Regala 2011).

A pesar de que en alguna de las publicaciones consultadas el número exacto de objetos de adorno no se compute (Moura y Aubry 1993) y de que la región portuguesa sea la menos prolífica en cuanto a este tipo de materiales, el número total de estos asciende a 117 elementos, el doble que los documentados, por ejemplo, en el Gravetiense (Álvarez Fernández y Avezuela Aristu 2012). En Portugal el 80\% de los objetos de adorno-colgantes están realizados sobre moluscos, le siguen aquellos realizados sobre hueso (16\%) y sobre dientes (4\%) (Fig. 2). Dentro de este porcentaje la evidencia mayor de moluscos perforados se concentra, sobre todo, en los yacimientos de Caldeirão y de Vale Boi con una presencia mayoritaria del gasterópodo marino L. obtusata. Este molusco también aparece en los niveles solutrenses de Anecrial (4), Lagar Velho (1), Gruta das Salemas (12) y Lapa de Rahína (1). Algunos de los ejemplares se encuentran sin perforar y su presencia en los yacimientos se interpreta como una posible reserva de materia prima para su posterior transformación en objeto de adorno-colgante. En el yacimiento de Vale Boi algunas de estas piezas se incluyen en la categoría de no perforadas aunque están fragmentadas posiblemente siendo ese el resultado de un proceso de fabricación fallido en el mismo yacimiento (Tátá Dos Anjos Regala 2011).
Otros gasterópodos marinos aparecen representados en el repertorio de Caldeirão (2 Nasarius reticulatus y 7 Aporrhais pespelescani) y de Vale Boi (6 Trivia arctica).

Es en estos dos mismos yacimientos donde encontramos los únicos testimonios de escafópodos, y también de gasterópodos fluviales (Theodoxus fluviatilis).

Las técnicas de manufactura no difieren en mucho respecto a otras zonas reduciéndose casi en exclusiva a las técnicas de presión y rotación. Excepto un ejemplar de Trivia arctica de Vale Boi biperforado todos los adornos cuentan con una sola perforación. En este mismo yacimiento las L.obtusata están perforadas con una técnica de presión directa desde el interior de la abertura menos dos ejemplares en los que el gesto se realiza desde el exterior de la concha. En cualquier caso lo que prima es la rapidez en la ejecución de la perforación y no el control de la fuerza para evitar posibles fracturas (Tátá Dos Anjos Regala 2011).

En el yacimiento de Caldeirão se documentan 19 falanges de ciervo con una perforación circular y otra ovalada en las caras interna y externa. A pesar del mal estado de conservación y de que gran parte de las piezas están completamente concrecionadas, estas se han interpretado como objetos de adorno-colgantes o marcadores étnicos exclusivos del yacimiento de Caldeirao dando un origen antrópico a las perforaciones (Zilhão 1997). Sin embargo un estudio tecnológico más profundo no permite desligar este tipo de perforaciones de las producidas por carnivoros ya que el estado de las piezas no impide discriminar entre un tipo $u$ otro de perforaciones (Chauvière 2002). Dos de los ejemplares están parcialmente cubiertos de un colorante rojo, lo cual podría inclinar levemente la balanza hacia la teoría del origen antrópico o en cualquier caso de un aprovechamiento oportunista de unas piezas ya perforadas.

En cuanto a los dientes, el porcentaje de adornos elaborados sobre estos es muy inferior y se limita a un escaso 4\% del total. Entre estos destacan los caninos atrofiados de ciervo perforados de Caldeirão y Buraca Grande; completa el conjunto un canino de lince de Lapa do Suão. Los dos caninos atrofiados de ciervo de Caldeirão presentan una preparación de la superficie por raspado-ranurado a modo de preparación y una perforación por rotación bipolar en uno de los casos. Ambas piezas tienen unas marcadas huellas de uso (Chauvière 2002).

\section{REGIÓN MEDITERRÁNEA}

Los datos recogidos para esta región proceden del estudio y revisión de los materiales de determinados yacimientos como la Cueva de Ambrosio, Santa Maira, Nerja (Tesis doctoral de B.A., en preparación), Reclau Viver y Cau de les Goges (Álvarez-Fernández 2002) así como de la información bibliográfica disponible sobre otros sitios como La Arbreda 


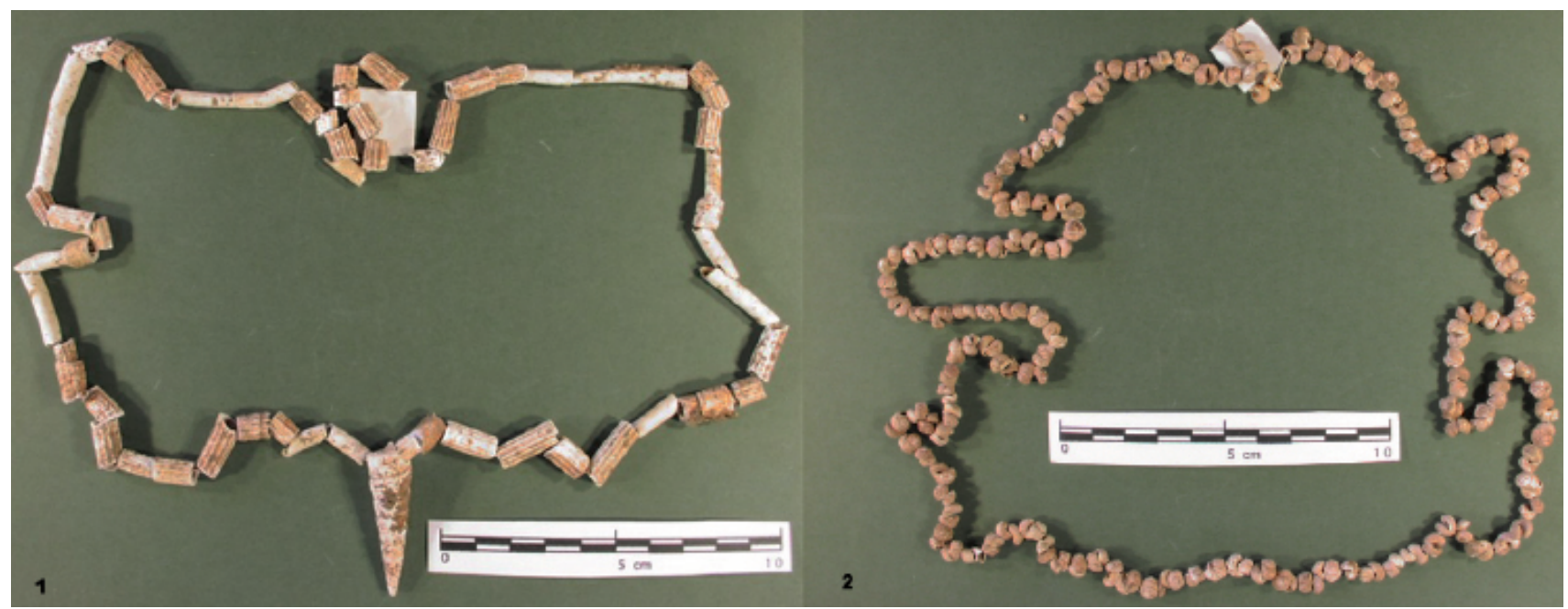

\ FiguRA 8. Reclau Viver, nivel F. 1. Collar de Dentalium sp y Turritela sp. 2. Collar de Homalopoma sanguineum (Foto: Museu Arqueologic de Banyoles).

(Soler i Masferrer y Maroto i Genover 1987), Parpalló y Cendres (Soler Mayor 2001), Beneito (Iturbe et al. 1993; Soler Mayor 2001), Volcán del Faro (Soler Mayor et al. 2013), La Boja (Zilhão et al. 2010), Tajo de Jorox (Simón Vallejo et al. 2006) y Cueva de los Ojos (Toro Moyano y Almohalla Gallego 1985). En algunos de estos casos la contabilización que se hace es aproximada porque en dichas publicaciones no se especifica el número de piezas (Soler i Masferrer y Maroto i Genover 1987; Zilhão et al. 2010). Los materiales proceden tanto de excavaciones antiguas (excavaciones de Corominas en Reclau Viver) como de excavaciones más recientes (Nerja, Cueva de Ambrosio) o de otras en las que todavía se está trabajando (Cova de Santa Maira).

En la región mediterránea el número de objetos de adorno-colgantes aumenta su número de manera importante a partir del periodo que nos ocupa. De este modo contabilizamos alrededor de 3000 elementos en 13 yacimientos (Fig. 1) frente a los escasos 150 documentados en el Gravetiense (Álvarez Fernández y Avezuela Aristu 2012).

Solo dos materias primas son las que se seleccionan para la elaboración de dichos elementos: las conchas de moluscos y los dientes. La más empleada son las conchas de moluscos, mayoritariamente marinos, y sobre todo gasterópodos y escafópodos. Los moluscos representan casi la totalidad de la muestra frente a 14 ejemplares de dientes perforados (Fig. 2).

Este aumento considerable en el número de adornoscolgantes respecto a épocas anteriores se debe en gran parte a que en el Solutrense medio de Reclau Viver (Gerona), se encuentra la mayor concentración de adornos-colgantes documentada en la Península Ibérica. Se trata de más de 2000 ejemplares de Homalopoma sanguineum junto a otras especies como Cyclope pellucida, Antalis sp., L. obtusata o Turritella sp. la mayor parte de ellos perforados (ÁlvarezFernández 2006).
Todos estos ejemplares se hallaron en las excavaciones arqueológicas llevadas a cabo en la galería de la cueva por Corominas en los años ' 40 del siglo XX donde gracias al desplome de la bóveda a finales del periodo Solutrense se conservó un yacimiento intacto. Con todos ellos, se realizó una reconstrucción museistica teórica y se confeccionaron una serie de collares, algunos de los cuales se encuentran hoy expuestos en el Museu Arqueològic de Banyoles (Fig. 8). Más de las tres cuartas partes de los gasterópodos proceden del Talud II, de la base del nivel F, donde Corominas describió en sus diarios cómo los ejemplares de $H$. sanguineum se encontraban unos junto a otros, incluso los ejemplares más pequeños dentro de la abertura de los de mayor tamaño y conservados así gracias a la precipitación de $\mathrm{CaCO}$. La mayor parte de estos ejemplares presentan huellas de pulido por uso en el contorno de sus perforaciones (ÁlvarezFernández 2002).

Después de $H$. sanguineum, los moluscos más abundantes son los escafópodos (13\% del total) con más de 390 ejemplares y presentes en 11 de los 13 yacimientos de la zona. En Parpalló, Cau de les Goges o Volcán del Faro estos moluscos tubulares alcanzan unos valores que integran casi la mitad de los conjuntos alcanzando los 75, 28 y 39 ejemplares respectivamente. Su abertura natural es el método de suspensión empleado y se documenta el serrado del extremo apical para el agrandamiento del orificio. En Volcán del Faro todos los individuos están fracturados por sus dos extremos y cuentan con distintos tamaños y distintos grados de facturación, esto quizás sea debido a su uso como piezas de un elemento compuesto (Soler Mayor et al. 2013).

A los escafópodos le sigue en número L. obtusata, con 90 ejemplares en Ambrosio y una treintena en Nerja (Fig. 10), mientras que se han documentado en un número mucho menor en Reclau Viver, Arbreda, Santa Maira, La Boja o Tajo de Jorox. 


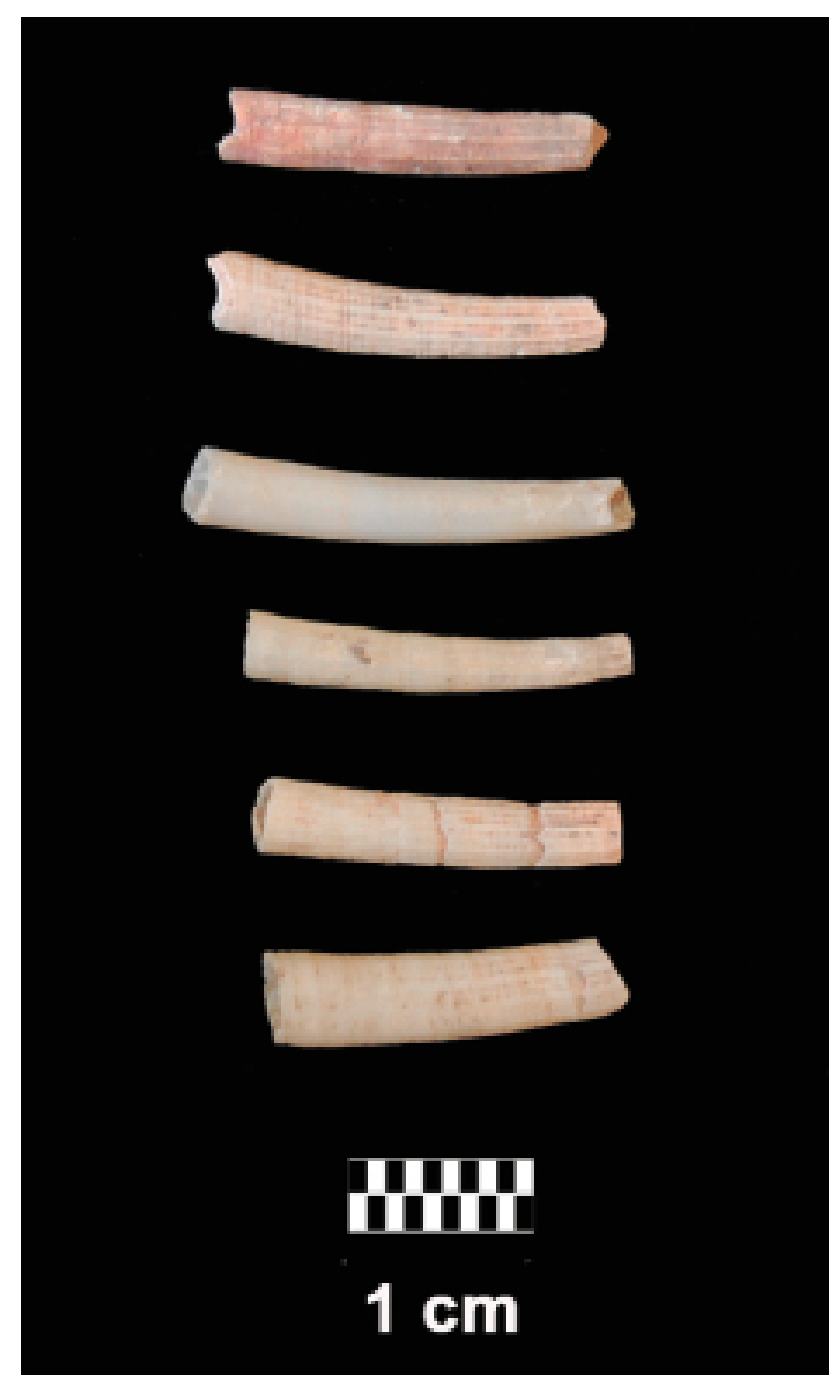

A Figura 9. Escafópodos. La Cueva de Ambrosio, microestratigrafía Solutrense.

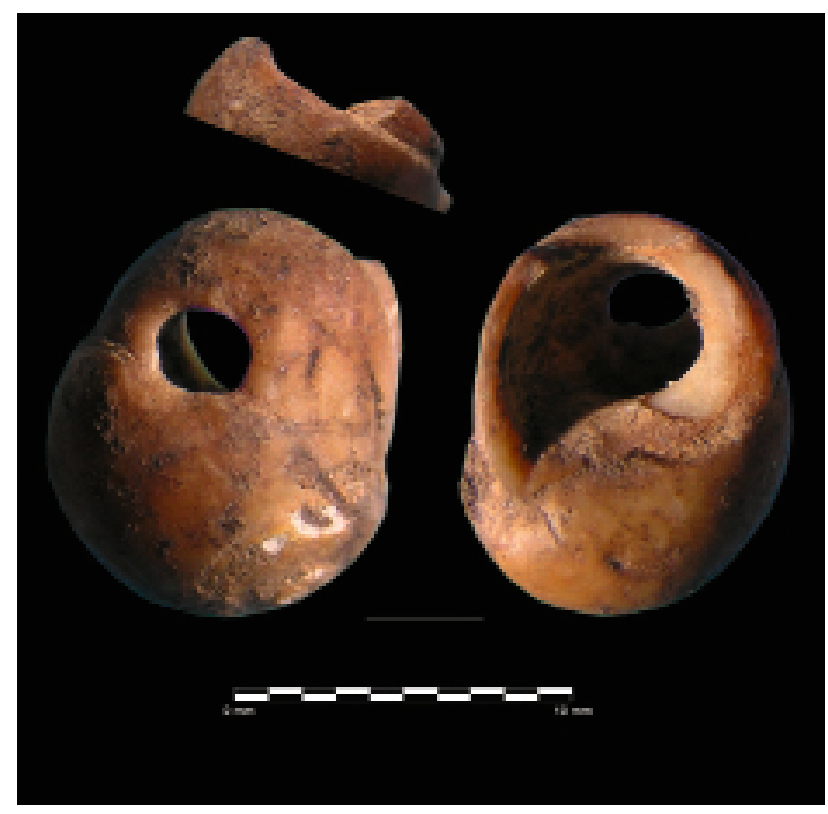

A Figura 10. Littorina obtusata perforada. La Cueva de Nerja, NV 8.
La Cueva de Ambrosio (Almería), Parpalló y Volcán del Faro (Valencia), Cendres (Alicante) o Nerja (Málaga) cuentan con la presencia de un número importante de ejemplares de adornos colgantes, casi todos ellos elaborados sobre moluscos: más de 300, 150, 85, 40 y 80 respectivamente). El número de especies de gasterópodos representadas también es mucho más elevado que en épocas anteriores y respecto a la región cantábrica y Portugal. Se han determinado más de una veintena de especies: Columbella rustica; Cyclope neritea; Cerithium vulgatum; $H$. sanguineum; Littorina saxatalis; Nassarius gibbosulus; $N$. incrassatus; Nassarius reticulatus; N. Iapillus; Patella caerulea; T. arctica, Turritella sp., entre otras. Cabe resaltar la presencia del gasterópodo dulceauicola Theodoxus fluviatilis,. En Cendres y en Ambrosio se contabilizan 30 y 55 ejemplares respectivamente. Algunos de los ejemplares de La Cueva de Ambrosio se encuentran sin modificar y su presencia en el yacimiento puede ser interpretada como una reserva de materia prima para su posterior transformación en objetos de adorno-colgantes. Dos ejemplares de otro gasterópodo fluvial (Melanopsis) proceden de Les Cendres.

En el caso de los bivalvos marinos su representación no es muy elevada ( $2 \%$ del total) comparada con los gasterópodos, aún así existen casi 60 ejemplares repartidos entre todos los yacimientos. Los sitios en los que se documentan mayor número de ejemplares son Volcán del Faro, Santa Maira y Ambrosio, el resto de testimonios se reducen a presencias puntuales de una sola pieza. En Volcán del Faro cabe resaltar que el número de bivalvos (si contabilizamos aquellos perforados y aquellos que, aun sin estarlo, su aportación al yacimiento solo puede responder a su posterior utilización como adornos) es superior al de gasterópodos (23 bivalvos frente a 4 gasterópodos), invirtiendo así la relación que existe en el resto de yacimientos mediterráneos. La especie predominante es Glycymeris sp., seguida de Pseudamussium sulcatum y Cerastoderma edule (Fig. 11) aunque también se encuentran otras especies como Acanthocardia tuberculata, - Pecten jacobeus. Las valvas perforadas cuentan con una sola perforación -al igual que los gasterópodos- situada a la altura del umbo. En alguno de los casos, el orificio no es fruto de la intervención antrópica, sino que ha sido producida por meteorización, rodamiento, organismos marinos $u$ otros moluscos. No obstante es importante remarcar que estos ejemplares han sido recogidos precisamente por poseer esa característica y la perforación cumple la misma función que una antrópica, permitir la suspensión, cosido o sujeción del objeto.

En la manufactura, tanto de gasterópodos como de bivalvos, se documentan diferentes técnicas -solas o combinadas- como la abrasión, la percusión, la presión, el serrado o la rotación sin que ninguna de ellas prevalezca sobre las otras. También se identifican incisiones previas a la perforación con el objetivo de fijar la punta lítica en algu- 


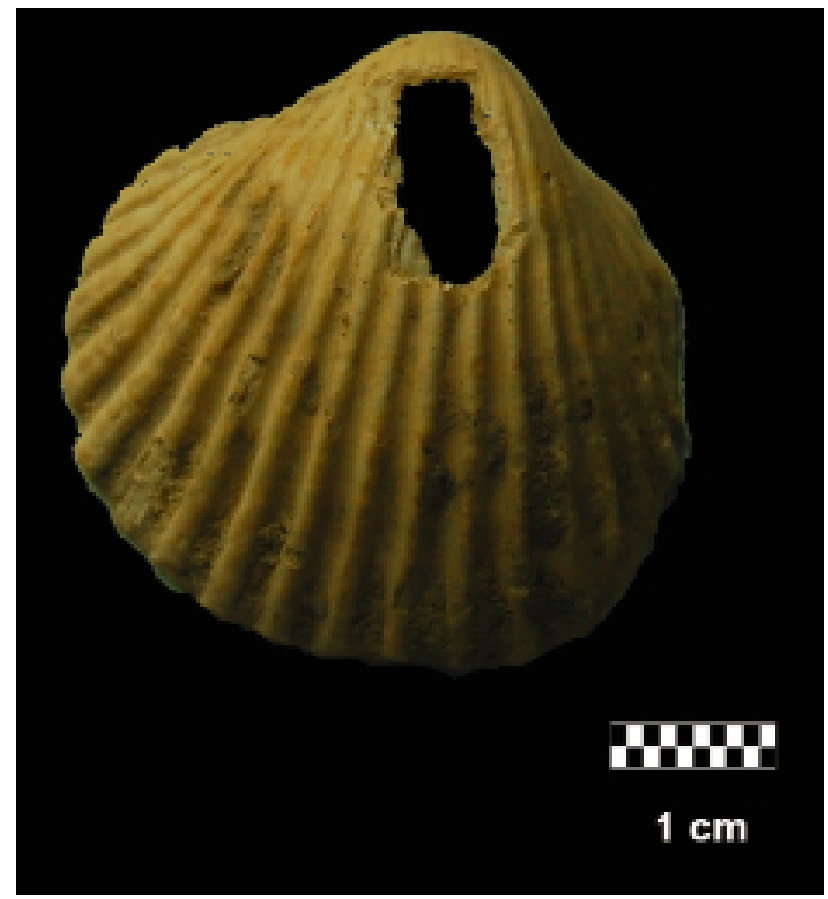

৯ Figura 11. Cerastoderma edule perforado. La Cueva de Santa Maira, Solutrense.

nos elementos de Volcán del Faro (Soler Mayor et al. 2013), reutilizaciones de moluscos en los que al realizar un primer intento de perforación el labio de la abertura ha resultado fracturado y se ha procedido a realizar una segunda perforación (ejemplares de L. obtusata de La Cueva de Ambrosio).

Otras materias primas como los dientes también se perforan, es el caso del canino de lince (Fig. 12) y los caninos de zorro perforados de La Cueva de Ambrosio o los caninos atrofiados de ciervo de Santa Maira (Fig. 13) y de Volcán del Faro. Todos los dientes transformados en adornos poseen una sola perforación situada a la altura de la raíz. La técnica escogida en la mayor parte de los casos es la rotación bipolar precedida de una preparación de la superficie.

En la mayoría de los yacimientos, en clara relación con estos elementos aparecen restos de colorante rojo, principalmente ocre (Álvarez-Fernández 2002; Soler Mayor 2001; Soler Mayor et al. 2013).

\section{CONCLUSIONES}

Durante el periodo Solutrense son numerosas las evidencias de objetos de adorno-colgantes que se documentan en los diferentes yacimientos de la Península Ibérica. Esto queda plasmado en un aumento del número de ejemplares en todas las zonas y en un mayor repertorio de las materias primas empleadas y de especies de moluscos seleccionados.

La zona más prolífica en cuanto a este tipo de elementos es la mediterránea, seguida de la cantábrica y de Portugal, aunque es en la región cantábrica donde se hace uso de

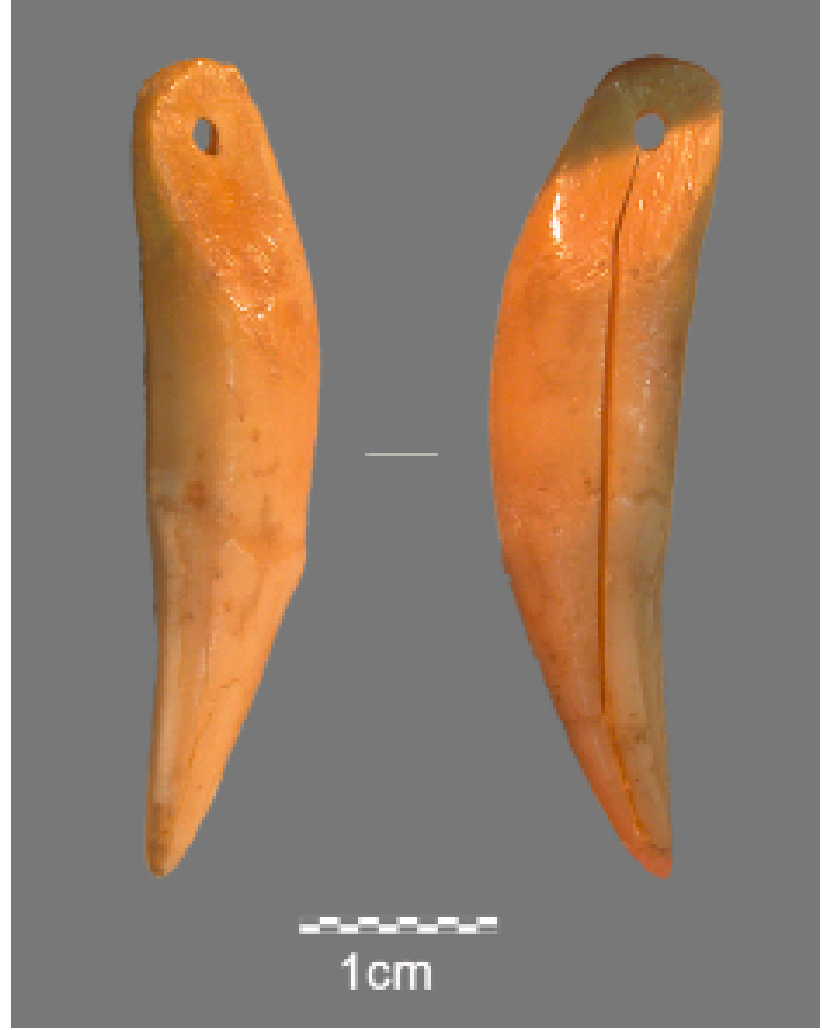

\ Figura 12. Canino de lince perforado. La Cueva de Ambrosio. Nivel VI.

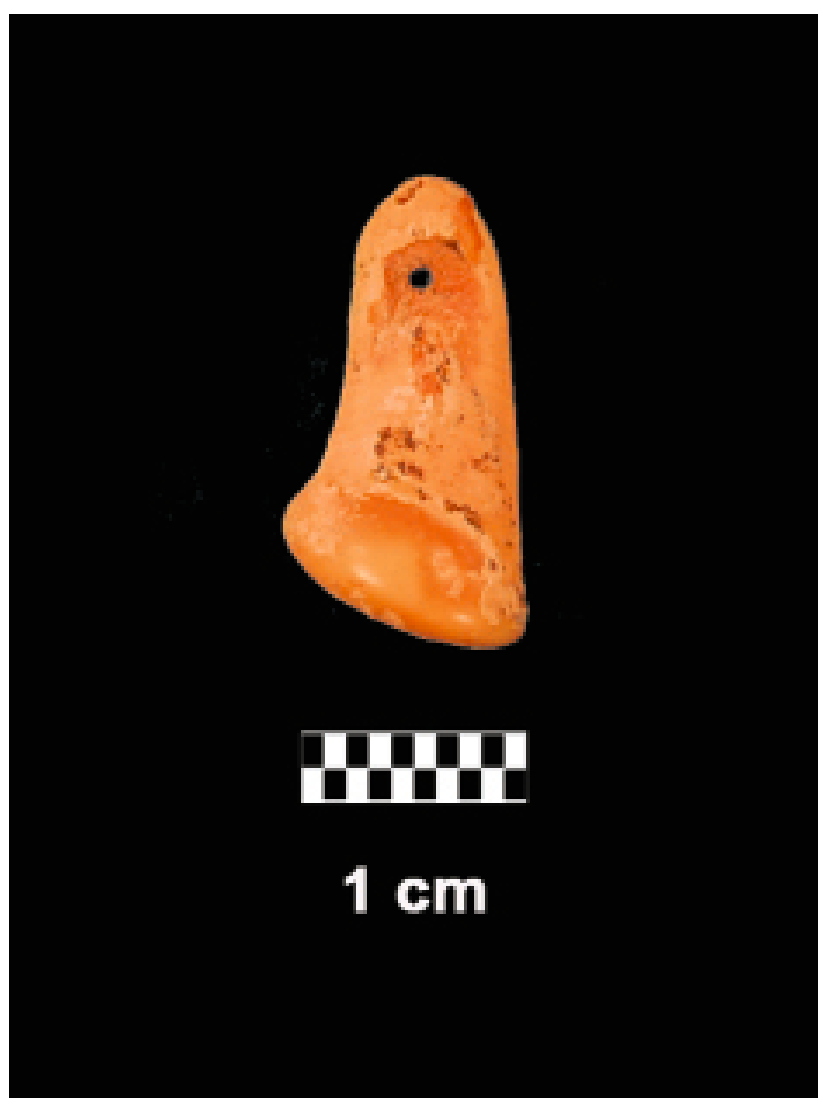

A Figura 13. Canino atrofiado de ciervo perforado. La Cova de Santa Maira, Solutrense. 
una selección más variada de materias primas. Las conchas de moluscos marinos (principalmente gasterópodos) son más abundantes en la región mediterránea y en Portugal y los dientes, en la región cantábrica.

En los yacimientos de la fachada atlántica (región cantábrica y Portugal) predomina el uso del gasterópodo marino Littorina obtusata -como en periodos anteriores-. En el Mediterráneo este lugar lo ocupa Homalopoma sanguineum gracias a la acumulación de Reclau Viver. En la región mediterránea cabe resaltar el uso de escafópodos durante este periodo en casi todos los yacimientos de la zona, siendo en algunos (Cau de les Goges, Parpalló, Santa Maira o Ambrosio) los moluscos más representados (Fig. 9).

En la región cantábrica los caninos atrofiados de ciervo perforados son los dientes más frecuentes en el registro solutrense. Aunque en un número inferior, lo mismo ocurre en Portugal (Buraca Grande) y en la fachada mediterránea (Santa Maira, Volcán del Faro). Los caninos de lince perforados también tienen su pequeña representación en las tres regiones (Altamira, Ambrosio y en Lapa do Suão).

Los adornos realizados a partir de otras materias primas (hueso, asta, marfil, minerales, ambar) no son muy abundantes y se concentran en la región cantábrica con la excepción de las falanges de ciervo de Caldeirão.

En cuanto a la manufactura se identifican diferentes tipos de técnicas para perforar dichas materias primas (abrasión, rotación, presión, percusión y serrado) y predominan las piezas con una sola perforación. Un gran número de estos elementos presentan huellas de uso alrededor de sus perforaciones (pulido, deformaciones o fracturas por el uso) así como en el resto de su superficie debido a la fricción.

En lo relativo a la proveniencia de estas materias primas, se trata de materiales locales recogidos cerca de los yacimientos -en el caso de los dientes procedentes de la caza o de carcasas abandonadas de animales muertos- 0 , en el caso de los moluscos, en la costa o en los cursos fluviales más próximos (en algunos casos las distancias son de $60 \mathrm{Km}$ como en La Cueva de Ambrosio o $45 \mathrm{Km}$ como Bolinkoba. Su análisis tafonómico muestra que muchas de ellas tienen marcas de abrasión en su superficie causadas por la acción del agua y mar y del entorno arenoso.

Conchas mediterráneas como Nassarius mutabilis aparecen en la región cantábrica en los yacimientos de El Ruso y se citan ejemplares en Cueto de la Mina (Álvarez-Fernández y Fernández 2011). Esto es una evidencia de que los cazadores recolectores de esta zona mantuvieron contactos con la zona mediterránea, en el caso de Cueto de la Mina estamos hablando de $600 \mathrm{~km}$.

Algunas especies consideradas tradicionalmente como atlánticas se documentan en la región mediterránea. N.lapilllus y L.obtusata y saxatalis en yacimientos como La Cueva de Ambrosio, Nerja o Santa Maira. Su presencia se puede explicar de dos maneras, ya sea por el contacto entre grupos o bien por la colonización de estas especies del oeste del mediterráneo en ciertos momentos del Máximo Glacial.

Es probable que parte de los Dentalium documentados en sitios del Mediterráneo (por ejemplo, de Reclau Viver) procedan de yacimientos geológicos. En el caso del marfil, su origen posiblemente tendría lugar en el intercambio con grupos de cazadores-recolectores provenientes de zonas más al norte del continente No descartamos, sin embargo, la posibilidad de que las defensas fueran aprovechadas en un estado sub-fósil.

Hay que indicar que en la región cantábrica, muchas de las piezas realizadas en dientes, hueso, asta y marfil aparecen grabadas con motivos figurativos lineares (sobre todo incisiones cortas profundas paralelas). No hay evidencias de decoraciones figurativas.

Además, y al igual que ocurre en otros periodos del Paleolítico superior, hay evidencia de la aplicación de colorantes en los objetos de adorno en todas las regiones. Posiblemente fueron coloreadas para resaltar sus formas (contraste de color entre la dentina y la raíz de los dientes) o la decoración de sus superficies, o bien se tiñeron al estar en contacto con superficies pintadas (piel humana, vestimentas, liana de suspensión).

Existe poca información sobre los contextos de los adornos ya que la mayor parte de las veces se trata de hallazgos aislados. Sin embargo en algunos casos contamos con más información acerca de la contextualización de estos objetos y se puede hablar de piezas que fueron encontradas juntas ya sea porque fueron depositadas a la vez intencionalmente, abandonadas, olvidadas o perdidas. El caso más excepcional es la acumulación de más de 2000 ejemplares de Homalopoma sanguineum perforados (junto a otras especies) en Reclau Viver. Acumulaciones de mucha menor envergadura se dan en Altamira, El Mirón, La Riera, Las Caldas o La Cueva de Ambrosio. En otros sitios también se documentan ejemplares sin perforar que pudieron formar parte de una reserva de materia prima, piezas fracturadas durante el proceso de fabricación (La Riera, Las Caldas, La Garma A, Vale Boi, La Cueva de Ambrosio) o en alguna fase de ese proceso (Las Caldas) lo cual junto a la identificación de las técnicas de perforación y las huellas de uso presentes en las perforaciones nos aporta más pistas sobre la cadena operativa de dichos elementos.

En definitiva, durante el Solutrense la continuidad respecto al periodo anterior (Gravetiense) en lo que respecta a las técnicas empleadas para transformar las materias primas en objetos de adorno-colgantes está contrarrestada por un incremento importante en cuanto al número de objetos de adorno-colgantes documentados en todas las regiones pero sobre todo en la mediterránea; las materias primas empleadas siguen siendo básicamente las mismas aunque el repertorio de especies de moluscos es más amplio y en la región 
cantábrica se hace uso de nuevos materiales. Todo esto ve su reflejo en un intercambio de objetos a corta y larga distancia que culminará en el periodo posterior, el Magdaleniense, momento del Paleolítico superior en el que se producirá una verdadera explosión documentándose gran variedad y número de adornos en casi todos los sitios arqueológicos. •

\section{REFERENCIAS BIBLIOGRÁFICAS}

Álvarez-FernÁndeZ, E. 2002: "Ejemplares perforados del gasterópodo Homalopoma sanguineum durante el Paleolítico superior en Europa occidental". Cypsela 14: 43-54.

- 2006: Los objetos de adorno-colgantes del Paleolítico superior y del Mesolítico en la Cornisa Cantábrica y en el Valle del Ebro: una visión europea. Universidad de Salamanca. Salamanca.

- 2010-2011: "Personal ornaments in Europe during the Solutrean: evidences from Cantabrian Spain)". Sautuola XVI-XVII: 45-52.

- 2010: "Una de cal y otra de arena: Primeras evidencias de explotación de moluscos marinos en la Península Ibérica". I Reunión Científica de Arqueomalacología de la Península Ibérica (León 20-21 mayo 2010). Férvedes: 95-103.

Álvarez-Fernández, E. y FernÁndez, R. 2011: "Marine Resources Exploitation in Cantabrian Spain during the Solutrean: molluscs, fish and marine mammals". Bulletin du Musée d'Anthropologie Préhistorique de Monaco 51: 87-97.

ÁlvareZ-FernándeZ, E. y JörIS, 0. 2007: "Personal Ornaments in the Early Upper Palaeolithic of Western Eurasia. An Evaluation of the record". Eurasian Prehistory 5 (2): 31-44.

Álvarez Fernández, E. y Avezuela Aristu, B. 2012: "Los objetos de adornocolgantes durante el Gravetiense en el SW de Europa". C. de las Heras et al. (Coord.). Coloquio Internacional el Gravetiense Cantábrico. Pensando el Gravetiense (Santillana del Mar, Cantabria 20-22 octubre 2011). Ministerio de Educación, Cultura y Deporte y Museo de Altamira. 593-603.

Bicho, N. F., Gibaja, J. F., Stiner, M. y Manne, T. 2010: «Le paléolithique supérieur au sud du Portugal: le site de Vale Boi». L'Anthropologie 114 (1): 48-67.

CHAUVIĖRE, F.-X. 2002: «Industries et parures sur matières dures animales du Paléolithique supérieur de la grotte de Caldeirâo (Tomar, Portugal)». Revista portuguesa de arqueologia. 5 (1): 5-28.

iturbe, G., Fumanal, M. P., Carrión, J. S., Cortell, E., Martínez, R., Guillem, P. M., Garralda, M. D. y Vandermeersch, B. 1993: "Cova Beneito (Muro, Alicante): Una perspectiva interdisciplinarn. Recerques del Museu d'Alcoi 11: 23-88.

Moura, M. H. y Aubry, T. 1994: "Paleolítico da Serra de Sicó». V. M. Oliveira Jorge (Coord.). $10^{\circ}$ Congresso de Arqueologia Peninsular (Porto 12-18 de Outubro 1993). Sociedade Portuguesa de Antropologia e Etnologia: 43-54.

Ripoll LóPEZ, S. 1998: La cueva de Ambrosio (Almería, Spain) y su posición cronoestratigráfica en el Mediterráneo occidental. BAR International Series. 462. Oxford.

Simón Vallejo, M. D., Navarrete Rodríguez, I., Cortés Sánchez, M., LozanoFrancisco, M. C. y Vera-Peláez, J. L. 2006: "Nuevos elementos simbólicos sobre soporte malacológico del Paleolítico Superior de la provincia de Málaga (Andalucía, España)". J. L. Sanchidrian Torti et al. (ed.). IVSimposio de Prehistoria Cueva de Nerja. La Cuenca Medi- terránea durante el Paleolítico superior. 38.000-10.000 años (Nerja 23-26 noviembre 2004). Fundación Cueva de Nerja: 366-378.

SOler I MASferrer, N. y Maroto I Genover, J. 1987: "Els nivells d'ocupació del Paleolitic Superior a la cova de l'Arbreda (Serinyà, Girona)". Cypsela VI: 221-228.

SOler MAYOR, B. 2001: "Adorno, imagen y comunicación". V. Villaverde (ed). De Neandertales a Cromañones. El inicio del poblamiento humano en las tierras valencianas. Universitat de València. Valencia: 367-376.

Soler Mayor, B., Tiffagom, M. y Aura Tortosa, J. E. 2013: "La Cova del Volcán del Faro (Cullera): més preguntas que respostes. Primeres dades sobre els elements ornamentals". A. Sanchis Serra y J. L. Pascual Benito (ed.). Animals i arqueologia hui. I Jornades d'arqueozoologia Museu de Prehistòria de València: 159-180.

TÁtÁ Dos Anjos Regala, F. J. 2011: Os adornos do Paleolítico superior de Bale Boi (Vila do Bispo - Algarve). Dissertaçao para a obtençao do grau do Mestre em Arqueologia. Universidade do Algarve. Portugal.

Tejero Cáceres, J. M. 2013: La explotación de las materias óseas en el Auriñaciense. Caracterización tecnoeconómica de las producciones del Paleolítico superior inicial en la Península Ibérica. Archaeopress. British Archaeological Reports. International Series 2469. Oxford.

Toro Moyano, I. y Almohalla Gallego, M. 1985: "Descubrimiento de industria del Paleolítico superior en la provincia de Granada. El yacimiento solutrense de la "Cueva de los Ojos" (Cozvíjar, Granada)". XVII Congreso Nacional de Arqueología (1983): 97-104.

VANHAEREN, M. y D'ERRico, F. 2002: "The body ornaments associated with the burial". J. Zilhao y E. Trinkaus (eds.). Portrait of the artist as a child. The gravettian human skeleton from the abrigo do lagar velho and its archaeological context. Trabalhos de arqueologia: 154-186.

Veiga Ferreira, O. D. y Roche, J. 1980: "Os elementos de adorno do Paleolítico superior de Portugal". Arqueologia 2: 7-11.

ZıLHÃ0, J. 2007: "The Emergence of Ornaments and Art: An Archaeological Perspective on the Origins of "Behavioral Modernity"". Journal of Archaeological Research 15 (1): 1-54.

- 1997: O Paleolítico superior da Estremadura portuguesa. Edições Colibri. Lisboa.

Zilhão, J., Angeluccl , D. E., Badal, E., lucena, A., Martín, I., Martínez, S., VILLAVERDE, V. y ZAPATA, J. 2010: "Dos abrigos del Paleolítico superior en Rambla Perea (Mula, Murcia)". J. Mangado (ed.). El Paleolítico superior peninsular. Novedades del siglo XXI. (Barcelona 27-29 enero 2010). Seminari d'Estudis i Recerques Prehistòriques, Universitat de Barcelona: 137-148.

Zilhão, J., Angeluccl , D. E., Badal-García, E., D'Errico, F., Daniel, F., Dayet, L., Douka, K., Highamg, T. F. G., Martínez-SÁnChez, M. J., Montes-BernárdeZ, R., Murcia-Mascarós, S., PéreZ-Sirvent, C., RoldánGarcía, C., Vanhaeren, M., Villaverde, V., Wood, R. y Zapata, J. 2010: "Symbolic use of marine shells and mineral pigments by lberian Neandertals". Proceedings of the National Academy of Sciences 3 (107). 1023-1028. 